\title{
Vaccination Vehicles for COVID-19 Immunization - China, 2021
}

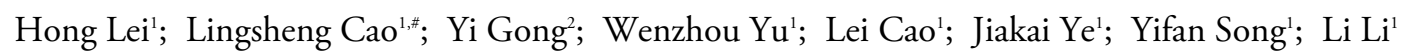

\begin{abstract}
Background: Recently, developed vaccination vehicles were repurposed and deployed for coronavirus disease 2019 (COVID-19) vaccination in China. We described the vehicles and reported an evaluation of vaccination throughput of these vehicles for COVID-19 vaccination in China.

Methods: We obtained daily reports of COVID-19 vaccine doses administered in vehicles in Hubei Province between March 16 and 29 of 2021. We determined the rate of COVID-19 vaccines given and evaluated the applicability of vaccination vehicles for COVID-19 vaccination.

Results: Vehicles with 2 vaccination stations are suitable for several real-world scenarios. Vehicles administered an average of 72 COVID-19 vaccine doses per hour, with an upper limit of 145 doses per hour.

Conclusion: Vaccination vehicles can save human and financial resources and provide high quality, effective, convenient, and rapid on-site vaccination services; they can increase the pace of COVID-19 vaccination.
\end{abstract}

\section{INTRODUCTION}

The global coronavirus disease 2019 (COVID-19) pandemic continues to be severe. Some of the emerging severe acute respiratory syndrome coronavirus 2 (SARS-CoV-2) variants of concern have shown increased transmissibility, challenging epidemic control and adding urgency to the deployment of COVID-19 vaccines. To rapidly build an immune barrier against SARS-CoV-2, many countries, including France (1), England (2), the United States (3), India (4), and China (5), are increasing the number of vaccination sites and building COVID-19 vaccination centers. However, building new vaccination centers is expensive and takes time. In China, in addition to using temporary vaccination clinics, specialized vaccination vehicles are being used to accelerate vaccination in communities, colleges, marketplaces, and companies.

China CDC proposed the development of vaccination vehicles in 2018. After completion of the design, production began in 2019, and in April 2020, the first vaccination vehicle was formally approved. The vehicles were developed to improve convenience, accessibility, and equity of access to vaccination services and promote timely information reporting from remote mountainous areas, settings that serve migrant children, and areas experiencing natural disasters.

In September 2020, an evaluation was conducted in 8 villages in Henan Province to assess the usefulness of vaccination vehicles for routine immunization of children in rural areas. The evaluation included questionnaire-based surveys for vaccination managers, staff, and parents, which measured their satisfaction with the vehicles. The evaluation showed that although some design features of the vehicles needed to be improved, such as the length of vaccination desks and the location of the refrigerator, the vehicle was well received by managers, staff, and parents and was useful for providing routine immunization services to rural children (G). A similar evaluation was conducted in Shandong Province in September and November of 2020 that showed that most adults were satisfied with the vaccination vehicle and thought it was very convenient (7).

As a result of the evaluations, the vehicles were improved by resizing vaccination desks and relocating the cold chain refrigerator. During the evaluations, several vaccination vehicles were beginning to be used for COVID-19 vaccination, including in settings that included government facilities, CDCs, harbors, prisons, and businesses in the provincial-level administrative divisions (PLADs) of Shandong, Shaanxi, Ningxia, and Xinjiang (8).

We conducted an evaluation of the vaccination vehicles for COVID-19 vaccination in several of these settings and report results of our evaluation. 


\section{METHODS}

\section{Vaccination Vehicle Description}

Vaccination vehicles are specialized vehicles equipped for storing, handling, administering vaccines, and treating immediate adverse reactions (e.g., allergic shock) following vaccination; they can be used for program and non-program vaccines. The vehicles have information systems that provide end-to-end data management. The information systems manage patient flow and support pre-inspection and registration of vaccinees, electronic notification, vaccine barcodescanning, recipient verification and confirmation, record of vaccine type, voucher-printing, and record of adverse reaction treatment (9).

The vehicles are 9.0 meters long, 2.5 meters wide, 3.7 meters high, and have 2 doors. They are decorated with green cartoon patterns and a tagline that highlights the National Immunization Program. A television and display screen are installed on the outside of the vehicle to show educational videos and to announce next persons to be served. The top of the vehicle is equipped with a collapsible 6 meters by 2.5 meters awning to shelter people from rain and sun. Vehicles are also equipped with solar panels that generate electricity for cold chain and vaccination refrigerators (Figure 1).

The vehicle's interior is two meters high with a registration area, two vaccination stations, a cold chain section, and an adverse reaction treatment area. The registration area is near the front door and has a desk with an electronic screen. A comfortable waiting bench with room for three people is attached to the registration desk. The cold chain area is located near the second door; the solar powered refrigerator is secured to the floor, which has a capacity of 100 liters and can maintain a temperature of $2{ }^{\circ} \mathrm{C}-8{ }^{\circ} \mathrm{C}$ for 7 days without external electrical power. The two vaccination stations are opposite the second door and the cold chain area; each has a vaccination desk and a countertop refrigerator. Countertop refrigerators store 200 vaccine vials or syringes in 8 drawers that open automatically via computer control and relay vaccine data to the data management system. The refrigerator displays vaccine storage temperature in real time and sends alarms for temperature excursions. The stations are equipped with printers, a medical waste disposal box, and a display screen for vaccination information. The adverse reaction treatment area is near the back of the vehicle and is equipped with a bed, an oxygen cylinder, a first-aid kit, and a privacy curtain. Each vehicle has an air conditioner, ultraviolet disinfection lamps, cameras, and a $5 \mathrm{G}$ network for connectivity. All devices can be controlled by speech (Figure 2).

\section{Vehicles in Use}

Vaccination vehicles are used by immunization clinics to conduct onsite vaccination in their respective jurisdictions. Vehicles are parked in accessible locations and vaccination workers from an immunization clinic can rapidly start providing services. People seeking vaccination scan a Quick Response (QR) code to get a wait-list number. All vaccination data are recorded in the Immunization Information System of the local immunization clinic and are submitted to the Immunization Information System of the provincial CDC and relayed to the National Immunization Information System of China CDC - all in near real time. After vaccination, there is a required 30 -minute observation period outside of the vehicle, after which

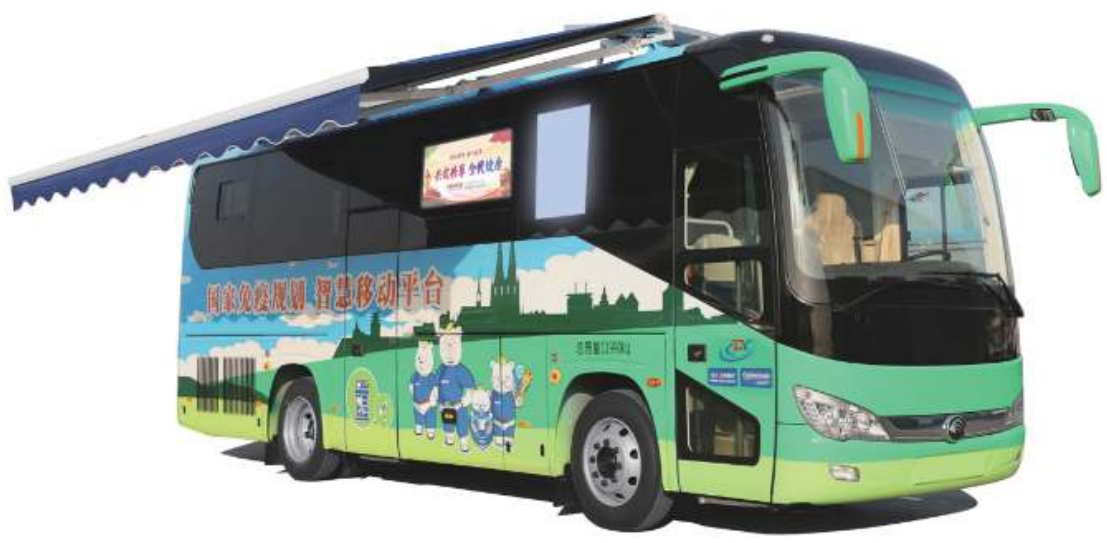

FIGURE 1. The exterior of a vaccination vehicle. 


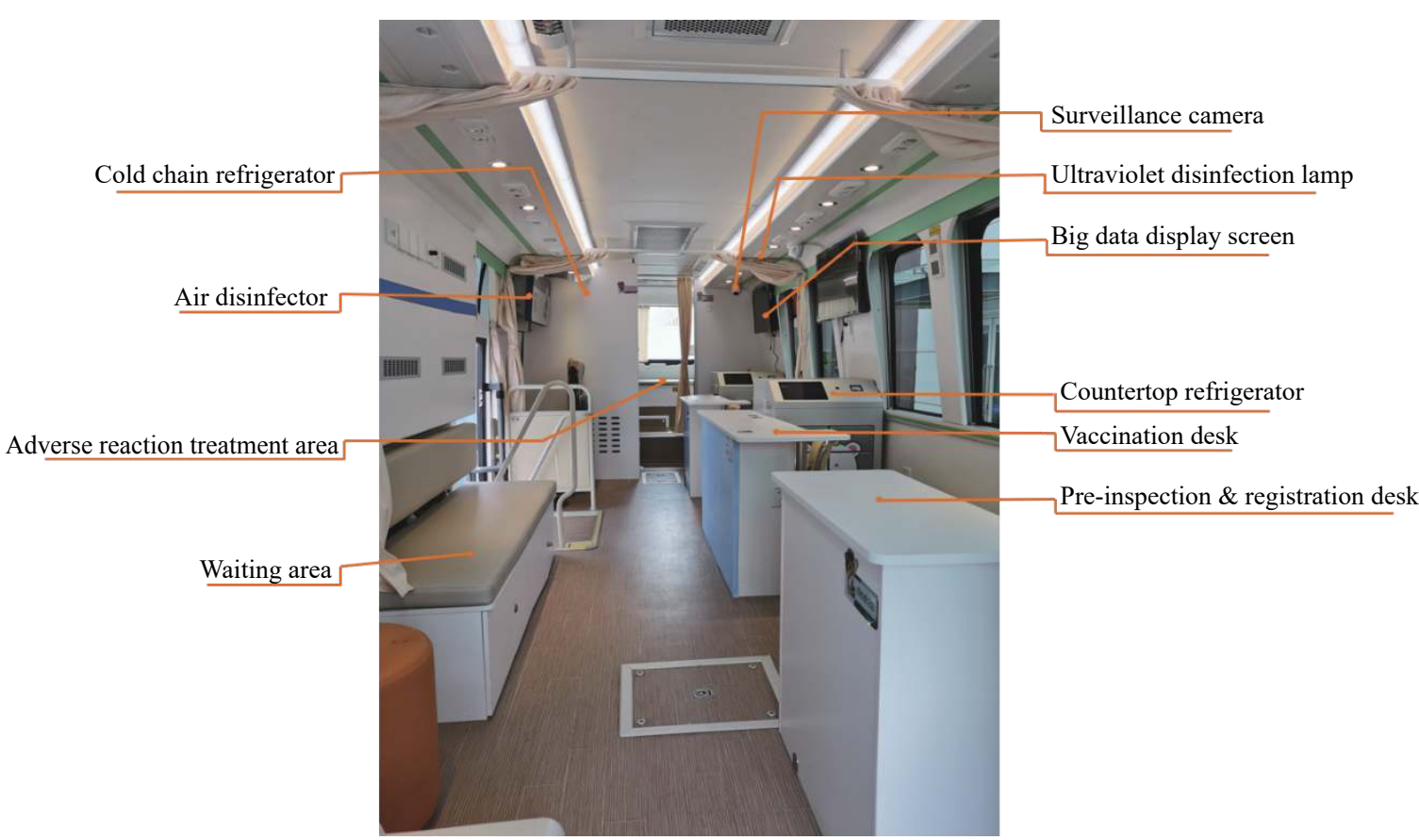

FIGURE 2. Design and layout of the vaccination vehicles.

Note: The vehicles have an air filtration unit and an ultraviolet disinfection lamp to keep the air clean. A display screen shows the vehicle's operating condition in real time. When an individual's turn for vaccination arrives, they enter one door, are registered and vaccinated, and they exit through the second door. In case of an adverse reaction, treatments can be provided in the vehicle while waiting for an ambulance.

time the vaccinated person can leave.

\section{Data Collection and Analysis}

In March 2021, Wuhan City of Hubei Province rented several vaccination vehicles to offer free COVID-19 vaccines in colleges, governments, prisons, businesses, and communities (10). We obtained daily reports of COVID-19 vaccination in vaccination vehicles in Hubei from March 16 to 29 of 2021; we used Microsoft Excel (version 2019, MS, Redmond, USA) to calculate the rate of vaccination.

\section{RESULTS}

During the study period, district CDCs and immunization clinics in Hubei Province rented 4 vaccination vehicles to provide vaccination services to adults in companies, hospitals, colleges, gymnasia, hotels, residential districts, public squares, and scenic spots. Staff in the vehicles administered 23,000 doses of COVID-19 vaccine; no serious adverse events occurred. Vehicle- 1 was used for 8 days and had a daily average vaccination rate that ranged from 30 doses an hour to 133 doses an hour; Vehicle-2 was used 11 days (28 to 117 doses per hour); Vehicle-3 was used 11 days
(19 to 114 doses per hour); and Vehicle 4 was used for 10 days (31 to 145 doses per hour) (Figure 3). The daily average vaccination rate across all vehicles was 72 doses per hour per vehicle, with a maximum of 145 doses per hour.

\section{DISCUSSION}

Our evaluation showed that one vaccination vehicle can provide up to 145 doses of COVID-19 vaccine in an hour. If this rate was sustained throughout the day, a single vehicle could potentially vaccinate up to 1,160 people in an eight-hour day. Based on this maximal throughput, the vehicles could be utilized more. Integrating the vehicles as outreach from immunization clinics and programs can promote progress on COVID-19 vaccination.

Vaccination vehicles represent a new service mode that complements fixed vaccination clinics. It can vaccinate in accordance with the Basic Functional Standards for Digital Vaccination Clinics, the Vaccination Work Specification, and the Vaccine Administration Law of the People's Republic of China. Vaccination vehicles are in mass production for deployment in China. Through early June of 2021, 

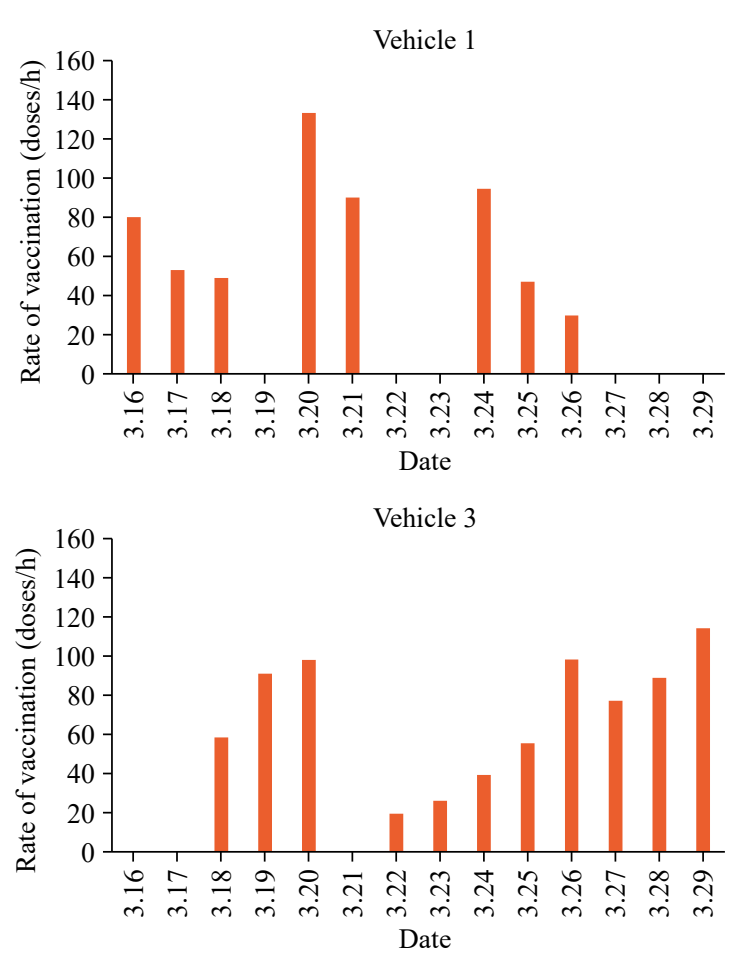
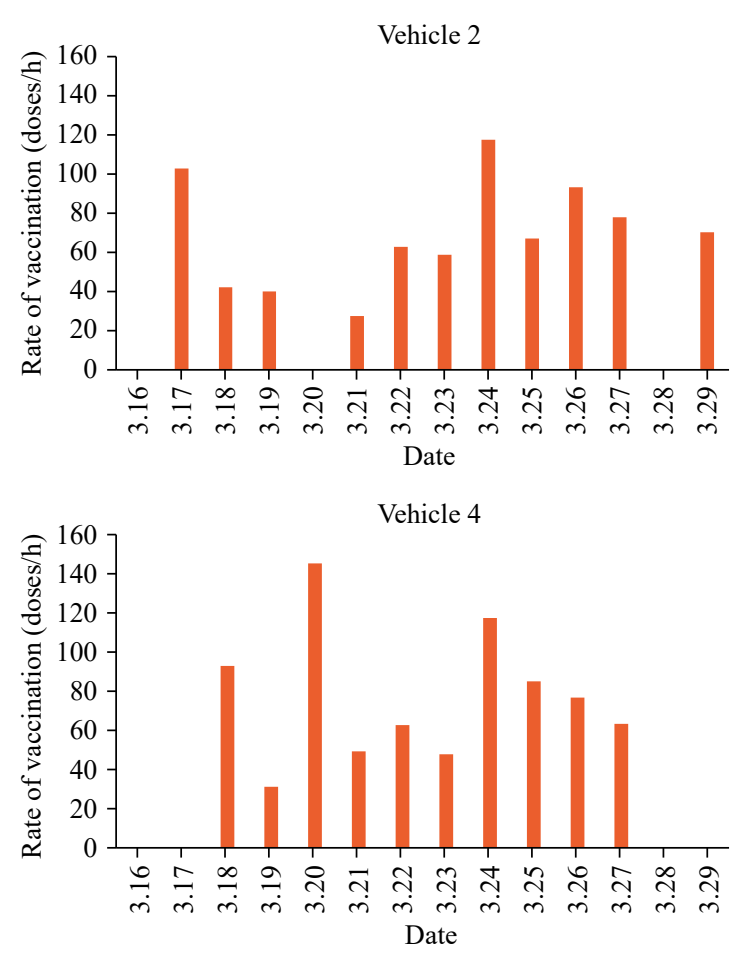

FIGURE 3. COVID-19 vaccine doses administered per day in vaccination vehicles 1-4, Hubei Province, March 16 to 29 , 2021.

140 vaccination vehicles were distributed around 21 PLADs and have collectively administrated 1 million doses of the COVID-19 vaccine. The Basic Guideline of the Function for Vaccination Vehicle was published in June 2021 (9) to ensure standardized production and use of the vehicles.

Vaccination vehicles improve convenience and timeliness of COVID-19 vaccination. They can be used in a variety of settings, including companies, markets, communities, and schools. For example, by bringing vaccinations to companies, people only need to step outside of the workplace and can quickly return to work after the 30 minutes observation period. By not having enclosed waiting rooms, vaccination vehicles may reduce the risk of respiratory infection. Onsite vaccination, with its greater convenience, may improve people's enthusiasm for vaccination and facilitate the vaccination effort.

Vaccination vehicles improve equity of access to COVID-19 vaccines and support timely data reporting. The new design and equipment are more advanced than many immunization clinics. Real-time data transmission and sharing allows every level of CDC to monitor progress in COVID-19 vaccination.

This study was subject to at least two limitations. First, the evaluation was conducted over a short time and the use of vaccination vehicles was still in their initial stages; further evaluation is warranted. Second, we only evaluated the rate of vaccination in the vehicles; a more comprehensive evaluation should be conducted.

Vaccination vehicles can save labor, material, and cost, while providing effective and equitable access to high quality immunization services in a variety of settings suitable for different populations and geographical conditions. Vaccination vehicles are important assets for COVID-19 vaccination in China and are contributing to the massive vaccination campaign in China. Other countries may want to use such vehicles in their COVID-19 campaigns.

Conflicts of interest: Authors declare that they have no known competing financial interests or personal relationships that could have appeared to influence the work reported in this paper.

Acknowledgements: The staff of Qingdao Haier Biomedical Company and Dr. Lance Rodewald.

doi: $10.46234 / \mathrm{ccdcw} 2021.178$

\# Corresponding author: Lingsheng Cao, caols@chinacdc.cn.

\footnotetext{
${ }^{1}$ National Immunization Program, Chinese Center for Disease Control and Prevention, Beijing, China; ${ }^{2}$ Qingdao Haier Biomedical Company, Qingdao, Shandong, China.
}

Submitted: June 26, 2021; Accepted: August 09, 2021 


\section{REFERENCES}

1. CCTV News. French Minister of Health: 35 large-scale vaccination centers for the COVID-19 vaccine will be built. 2021. https://mbd. baidu.com/newspage/data/landingsuper?context $=\% 7 \mathrm{~B} \% 22$ nid $\% 22 \% 3 \mathrm{~A}$ $\% 22$ news_10281207629977433544\%22\%7D\&ntype $=1 \&$ prom $=4$. [2021-3-23]. (In Chinese).

2. Guangming Net. The UK releases the COVID-19 vaccination plan: over 2.2 million people in the UK have received the first dose of the COVID-19 vaccine. 2021. https://m.gmw.cn/baijia/2021-01/12/ 1302028243.html. [2021-1-12]. (In Chinese).

3. Xinhua News Agency Client. United States: add COVID-19 vaccination sites. 2021. https://baijiahao.baidu.com/s?id=1690911064 216012077\&wfrspider\&forpc. [2021-2-6]. (In Chinese).

4. Russian Satellite News Agency. Russian Satellite News Agency, New Delhi, January 14. The Times of India quoted government officials' speeches and reported that India will start vaccinating COVID-19 at 3, 000 locations on January 16, and will expand to 5, 000 locations at the end of the month. 2021. http://sputniknews.cn/society/202101141032 884958/. [2021-1-14]. (In Chinese).

5. This is Xiangtan. The largest COVID-19 vaccine "square cabin vaccination site" in the province will be built in Xiangtan. 2021.
https://mp.weixin.qq.com/s/ebiFGGtoEIiY0-WT-T0UrA.

[2021-324]. (In Chinese).

6. Lei H, Cao LS, Wang CS, Yuan YL, Ren LG. Satisfaction with a mobile vaccination vehicle for rural children. Chin J Vaccin Immun 2021;27(2):202 - 5. http://dx.doi.org/10.19914/j.cjvi.2021032. (In Chinese).

7. Lei H, Cao LS. Satisfaction with a mobile vaccination vehicle for adult vaccination services. Chin J Vaccin Immun 2021;27(3):285-8. http://dx.doi.org/10.19914/j.cjvi.2021052. (In Chinese).

8. Sina Finance. The COVID-19 vaccine should be exhausted vaccination vehicle has been put into use. 2021. https://baijiahao.baidu.com/s?id= 1694038429080409925\&wfrspider\&forpc. [2021-3-12]. (In Chinese).

9. Chinese Preventive Medicine Association. Chinese preventive medicine association group standard announcement. 2021. http://www.cpma.org. cn/zhyfyxh/tzgg/202106/d537cd527f264da3ada9df5f63ce89d5.shtml. [2021-6-3]. (In Chinese)

10. Wuhan Learning Platform. Wuhan launches vaccination mode vaccination vehicle "door-to-door service". 2021. https://article.xuexi.cn/ articles/index.html?art_id=13262408370319897371\&t161622456827 2\&showmen=false\&studystyle_id=video_default\&sourceshare\&shareto $=w x \_$single $\&$ itemid $=13262408370319897371 \&$ refread_id $=8744 f 47 \mathrm{a}$ 967d-4a5c-a300-e2ccf1a23eee_1616410885951. [2021-3-20]. (In Chinese). 